\title{
QUISTE ODONTOGÉNICO EPITELIAL CALCIFICANTE EN ADOLESCENTE. REPORTE DE CASO CLÍNICO.
}

Calcifying Epithelial Odontogenic Cyst in an adolescent. Clinical Case Report

Fecha de Recepción: 10 de noviembre 2020
Cisto Odontogênico Epitelial Calcificante em Adolescente. Relato de Caso Clínico

Aceptado para su publicación: 30 de noviembre 2020

\author{
Autores: \\ Roque O Rosende1,2,a \\ Carlos A Rosales1,2,b \\ Patricia N Pavón Zarza1,2,c \\ Sebastián Krupp1,2,c
}

1. Carrera de Especialización en Cirugía y Traumatología Buco Maxilofacial. Facultad de Odontología. Universidad Nacional del Nordeste. República Argentina.

2. Módulo Patología y Diagnóstico I, II, III. Facultad de

Odontología. Universidad Nacional del Nordeste.

República Argentina.

a. Doctor de la Universidad Nacional del Nordeste en

Odontología (UNNE)

b. Magister en Gestión de la Salud Pública con Orientación

en Prácticas Preventivas (UNNE)

c. Odontóloga, Residente de la Carrera de Cirugía

y Traumatología BMF (UNNE)

d. Especialista en Cirugía y Traumatología

Bucomáxilofacial (UCA)

Correspondencia:

Rosales, Carlos Alejandro

Av. Libertad 5450, W3400 Corrientes

+540379445-7992

Correo electrónico:

rosalesale@live.com.ar

carosales@odn.unne.edu.ar

Conflicto de intereses:

los autores declaran no tener conflictos de interés.

Fuente de financiamiento:

autofinanciado.

\section{Resumen}

El Quiste Odontogénico Calcificante forma parte del grupo de Quistes Odontógenicos y No Odontogénicos del Desarrollo, según la Clasificación de Quistes Maxilares de la Organización Mundial de la Salud, 2017. Es una patología poco común sin predilección por el género, afectando tanto a la población joven como adulta involucrando por igual a mandíbula y maxilar y es frecuentemente encontrado en asociación con piezas dentarias incluidas. El objetivo de esta presentación fue diagnosticar y desarrollar un plan de tratamiento quirúrgico, acorde al caso clínico. Se reporta un caso clínico de paciente de género masculino de 15 años de edad, con presencia de una lesión quística de 5 meses de evolución en zona anterior del maxilar superior izquierdo. El diagnóstico histopatológico previa biopsia incisional se correlacionó con los hallazgos clínicos y radiográficos, lo cual revela en el estudio histopatológico la presencia de Quiste Odontogenico Epitelial Calcificante asociado a Quiste Dentigero. El tratamiento consistió en la remoción quirúrgica del quiste bajo anestesia general. Posterior a la enucleación se hicieron los controles postoperatorios y el seguimiento del paciente por un periodo de 2 años. La realización de una historia clínica exhaustiva, confirmación diagnóstica mediante biopsia y técnica quirúrgica correcta en la eliminación del quiste nos permitió obtener un resultado favorable.

Palabras clave: quiste odontogénico calcificado, quiste dentígero, adolescente, cirugía bucal (fuente: DeCS BIREME).

\section{Abstract \\ The Calcifying Dentistry Cyst is part of the group of Developmental Dentistry and Non-Dentistry Cysts, according to the Classification of Maxillary Cysts of the World Health Organization, 2017. It is a rare patholo}


gy without gender predilection, affecting both young and adult population involving equally the mandible and maxilla and is frequently found in association with included dental pieces. The objective of this presentation was to diagnose and develop a surgical treatment plan, according to the clinical case. A clinical case of a 15-year-old male patient is reported, with the presence of a 5-month-old cystic lesion in the anterior area of the left upper jaw. Histopathological diagnosis after incisional biopsy was correlated with clinical and radiographic findings, which reveals in the histopathological study the presence of Calcifying Epithelial Dental Cyst associated with Dentigerous Cyst. The treatment consisted of surgical removal of the cyst under general anesthesia. After enucleation, postoperative controls and follow-up of the patient were made for a period of 2 years. An exhaustive clinical history, diagnostic confirmation by biopsy and correct surgical technique in the elimination of the cyst allowed us to obtain a favorable result.

Key words: women's health, adolescents, health vulnerability, community-institutional relations (source: MeSH NLM).

\section{Resumo}

O Cisto Odontogênico Calcificante faz parte do grupo dos Cistos Odontogênicos e Não Odontogênicos de Desenvolvimento, segundo a Classificação de Cistos Maxilares da Organização Mundial da Saúde, 2017. É uma rara patologia sem predileção por gênero, afetando a população jovem como a população adulta, envolve a mandíbula e a maxila da mesma forma é frequentemente encontrado em associação com dentes incluídos. O objetivo desta apresentação foi diagnosticar e desenvolver um plano de tratamento cirúrgico, de acordo com o caso clínico. É relatado o caso clínico de um paciente de 15 anos de idade, masculino, com presença de lesão cística de 5 meses em região anterior de maxila superior esquerda. O diagnóstico histopatológico prévio à biópsia incisional foi correlacionado com os achados clínicos e radiográficos, o que revela no estudo histopatológico a presença de Cisto Odontogênico Epitelial Calcificante associado a Cisto Dentígero. O tratamento consistiu na remoção cirúrgica do cisto sob anestesia geral. Após a enucleação, os controles pós-operatórios e o acompanhamento dos pacientes foram realizados por um período de 2 anos. O preenchimento de uma história clínica exaustiva, a confirmação diagnóstica por biópsia e a técnica cirúrgica correta na retirada do cisto nos permitiram obter um resultado favorável.

Palavras-chave: cisto odontogênico calcificante, cisto dentígero, adolescente, cirugia bucal (fonte: DeCS BIREME).

\section{Introducción}

El Quiste Odontogénico Calcificante (QOC) es una lesión quística no neoplásica cuyo revestimiento epitelial presenta una capa basal bien definida de células cilíndricas, sobre la cual se dispone otra capa constituida con frecuencia por muchas células superpuestas y que puede parecerse al retículo estrellado, así como masas de células epiteliales fantasmas que pueden situarse en la barrera quística epitelial o en la cápsula fibrosa. Estas células fantasmas pueden calcificarse ${ }^{1}$. Fue descrito por primera vez por Gorlin, en 1962, reclasificado en el 2005 por la OMS como Tumor Odontogénico Quístico calcificante (TOQC). Sin embargo, en la última edición (cuarta) del año 2017 en la clasificación de tumores de cabeza y cuello de la OMS se lo clasifica nuevamente como un quiste re nombrándolo como Quiste Odontogénico Calcificante (QOC), y a la contraparte neoplásica, como Tumor Dentinogénico de Células fantasma $(T D C F)^{2}$. Es decir, reintegra al quiste odontogénico calcificante a la clasificación de QO y rechaza la terminología anterior de tumor odontogénico quístico calcificante que pretendían sugerir que son verdaderas neoplasias.

Es una patología poco común constituyendo el $0,37 \%$ a $2,1 \%$ de todos los quistes y tumores odontogénicos. El 65\% de los QOC se presentan en la región de incisivo y aproximadamente $20 \%$ está asociada con un odontoma.

Esta lesión no tiene predilección por ningún género afectando a pacientes entre los 5 a los 92 años y pueden tener una localización extraósea o intraósea, tanto en maxilar como en mandíbula, generalmente en la región anterior.

Clínicamente el Quiste de Gorlin se manifiesta como una lesión no neoplásica, indoloro, que causa la expansión de la corteza ósea y puede exhibir perforación de la placa ósea y en el examen radiográfi- 
co, es principalmente, lesión unilocular radiolúcida bien definida, aunque ocasionalmente multicolular ${ }^{3}$.

El examen clínico, histopatológico e imagenológico determina el tratamiento a seguir. La tomografía computada (dental scam) es un examen de fácil obtención, costo accesible, que permite la clara visualización tridimensional de las lesiones intraóseas, lo que facilita el planeamiento y la ejecución del tratamiento quirúrgico propuesto, evitando complicaciones y secuelas en el acto operatorio y en el posoperatorio ${ }^{4}$, y de esta forma permite el estudio de la lesión en corte axiales, sagitales y coronales además de la gran utilidad de una radiografía panorámica.

El propósito de una biopsia es determinar la naturaleza de la lesión, establecer un pronóstico y poder formular tratamientos específicos, su reporte, constituye un documento de valor médico-legal. La biopsia incisional es el procedimiento quirúrgico que consiste en la toma de una parte representativa de una lesión para someterla a estudio histopatológico.

Con relación al diagnóstico diferencial, existen varias entidades que pueden asociarse a esta lesión, tales como: el quiste dentígero, el tumor odontogénico adenomatoide, el odontoma compuesto, el tumor odontogénico epitelial calcificante, y el queratoquiste.

El tratamiento se limita a la enucleación quirúrgica de la lesión, dada la existencia de baja tasa de recurrencia con pronóstico favorable. El objetivo de esta presentación fue diagnosticar y desarrollar un plan de tratamiento quirúrgico, acorde al caso clínico.

\section{Caso Clínico}

Paciente de género masculino de 15 años de edad, que acude a la consulta en el Servicio de $\mathrm{Ci}$ rugía y Traumatología Buco Maxilofacial del Hospital Universitario de la Universidad Nacional del Nordeste, Provincia de Corrientes, por extrusión de la pieza dentaria 2.1 y leve aumento de volumen en la zona anterosuperior. Al interrogatorio, el paciente refiere que la lesión tuvo inicio hace aproximadamente meses atrás, siendo ésta de pequeña y que con el trascurso del tiempo fue aumentando de tamaño. Comentó haber presentado trauma en la zona hace aproximadamente dos semanas sin cambios. Se confeccionó la historia clínica completa y solicitud de examen imagenológico. Al examen clínico extraoral, presentó simetría facial. En la inspección intraoral, se observó mucosa de coloración normal, aumento de volumen de zona anterosuperior del lado izquierdo de aspecto tumoral por vestibular, plano oclusal alterado por la extrusión de la pieza dentaria 2.1, sin dolor. En la región palatina, se observó aumento de volumen. A la palpación fue de consistencia blanda y fluctuante.

Al estudio imagenológico de una tomografía de haz cónico, cortes axiales, sagitales, y coronales, se observó lesión osteolítica hipodensa, de forma unilocular, de márgenes bien definidos que envolvía a piezas dentarias 2.1, 2.2, 2.3 del maxilar superior, y desplaza las raíces de piezas 2.2 y 2.3 , de aproximadamente $2 \mathrm{~cm}$ de diámetro total sin desplazamiento del seno maxilar. En la región palatina, ocupaba la porción anterior derecha del paladar duro (Figura 1). En la ortopantomografía, se identificó una lesión radiolúcida que involucraba a piezas dentarias ya mencionadas (Figura 2).

Se confeccionó de manera completa la Historia Clínica, con firma del consentimiento informado. No refirió antecedentes médicos, personales u familiares de compromiso general. Se solicitaron estudios de laboratorio, cuyos valores estuvieron en rangos de normalidad. El diagnostico presuntivo fue de quiste dentígero, quiste radicular.

Para un diagnóstico de certeza, se procedió a realizar la biopsia incisional. Bajo técnica anestésica local se realizó una incisión lineal a 3 milímetros del borde libre de la encía con dos descargas verticales, una a nivel distal de la pieza dentaria 1.1 y otra descarga en distal de la pieza dentaria 2.2, obteniendo así un colgajo trapezoidal mucoperióstico. Se realizó osteotomía y se accedió a la lesión. Se tomó un fragmento de la misma y además se realizó la punzoaspiración con aguja y jeringa obteniendo un contenido de $5 \mathrm{ml}$ de color hemático. Para finalizar el procedimiento, se realizó la hemostasia con reposición del colgajo, sutura de los bordes de la herida con puntos simples y colocación de apósito compresivo. El informe histopatológico fue de Quiste Odontogénico 
Epitelial Calcificante asociado a Quiste Dentígero, realizado en el Servicio de Anatomía Patológica de la FOUNNE (Protocolo Número 19-003).

Una vez obtenido el diagnóstico definitivo, se decidió, en un segundo tiempo quirúrgico, la realización de biopsia escisional para la eliminación de la lesión.

Posteriormente se derivó al paciente, para una interconsulta con el Servicio de Endodoncia de la FOUNNE, para la evaluación y plan de tratamiento a seguir de las piezas implicadas en dicha patología.

En un segundo tiempo quirúrgico, se realizó la enucleación de la lesión. El paciente en decúbito dorsal bajo anestesia general con intubación nasotraqueal, antisepsia, colocación del campo según técnica, anestésica infiltrativa local al nervio alveolar anterior y cierre de circuito al nervio nasopalatino. Se realizó la incisión lineal, a nivel del fondo del surco desde la pieza dentaria 1.1, salvando el frenillo hasta la pieza dentaria 2.4 , se obtuvo un colgajo mucoperióstico de espesor total, se completó la osteotomía de la cortical ósea vestibular de la porción fenestrada con fresa número 6 , micromotor baja velocidad e irrigación con solución fisiológica, se realizó la enucleación con técnica de arrastre con gasa y cureta acodada para desprender la membrana quística en contacto con la superficie ósea remanente. Se realizó el control de la hemostasia y reposición del colgajo con sutura simple y

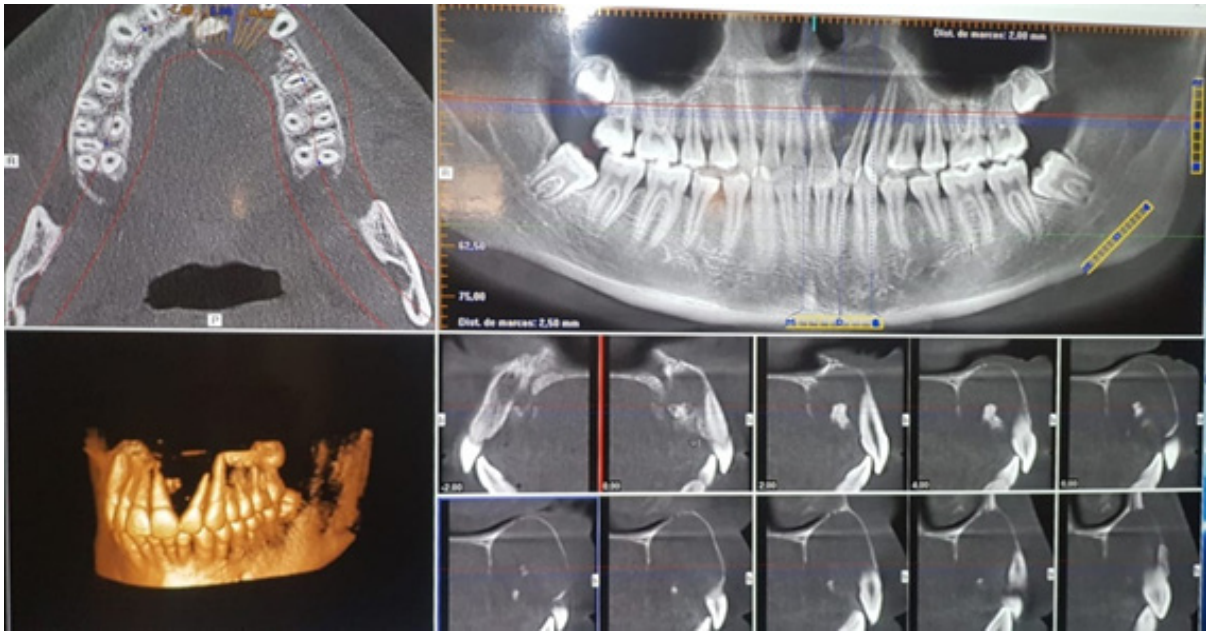

Figura 1. Tomografía computada con reconstrucción 3D en donde se observa una lesión quística con presencia de estructura calcificada.

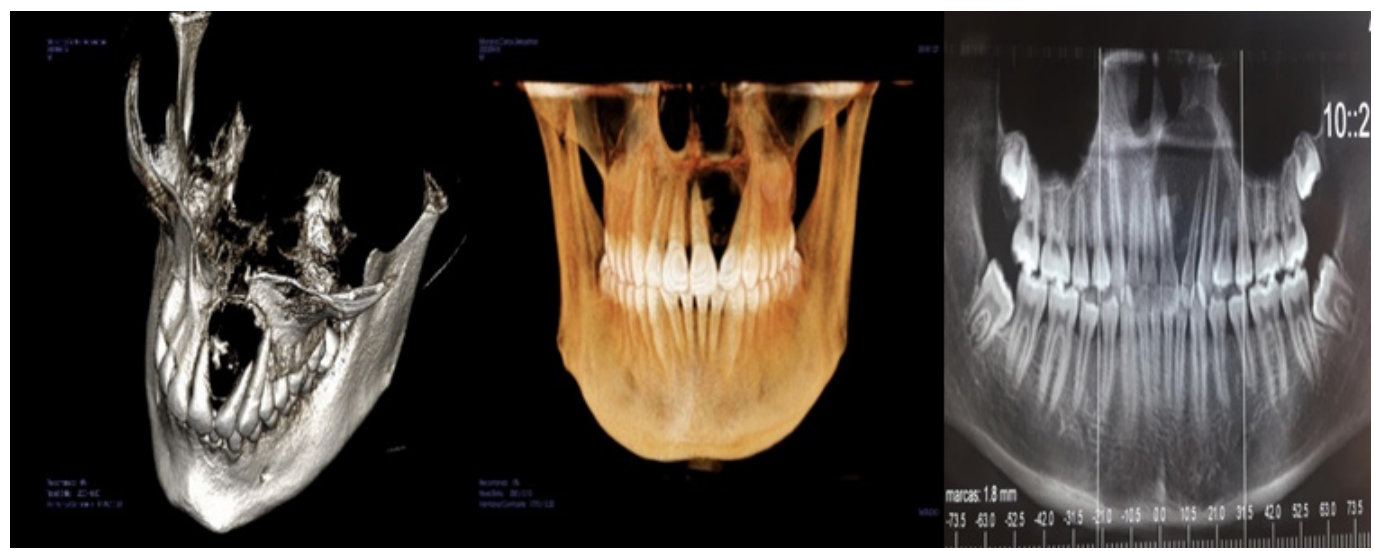

Figura 2. Tomografía computada con reconstrucción 3D en donde se observa una lesión quística con presencia de estructura calcificada. 
apósito compresivo. El procedimiento se realizó sin complicaciones. El control postquirúrgico y retiro de los puntos fue a los 7 días. Se realizaron controles clínicos e imagenológicos cada 6 meses durante el primer y segundo año de evolución, notándose en esta última, una neoformación ósea, de evolución favorable sin sintomatología.

En el último control imagenológico, se puede observar una evidente neoformación ósea en el sitio donde se ubicaba la lesión, así también se puede observar los tratamientos de conductos que se realizaron en las piezas dentarias involucradas (Figura 3).

\section{Discusión}

En 1981, Praestorius et al, publicaron una clasificación propia, dividiendo al QOC en dos entidades: un quise y una neoplasia. La entidad quística fue clasificada en tres tipos: 1) uniquístico simple, 2) uniquístico productor de odontoma, 3) uniquístico ameloblastomatoso. Desde su identificación en 1962, el QOC se presenta como una lesión central (intraósea) dentro de la mandíbula o maxilar, pero también puede ocurrir como periférica (extraósea) 5 . Las lesiones no tienen características radiológicas específicas, aunque con gran frecuencia presentan el aspecto de imágenes radiolúcidas uní o multiloculares bien circunscritas que contiene manchas radiopacas difusas que pueden asociarse incluso, a odontomas o a un diente no erupcionado ${ }^{6}$. En este caso clínico podemos observar la presencia de una estructura calcificante ubicada entre piezas dentarias 21 y 22.

Debido a la reciente clasificación, tras nuestra búsqueda bibliográfica encontramos que muchos artículos hacen referencia a Tumor Odontogénico Epitelial Calcificante, como Quiste Odontogénico Calcificante, tratándolas como una misma entidad patológica. Sin embargo, bibliografía anterior al año 2005 son de gran valor ya que describen de forma precisa esta patología bajo el término de Quiste de Gorlin.

Numerosas terminologías, dificultaron la búsqueda como: Tumor Odontogeno Quístico Calcificante, Adamantinoma atípico, Tumor Odontogenico Mixto, Colesteatoma. Estos términos reflejan la diversidad histopatológica de la entidad y confusión acerca de su naturaleza.

De acuerdo a los datos recogidos de la clínica y el estudio radiográfico realizado al paciente, se establecieron como diagnósticos diferenciales las mismas patologías que numerosas bibliografías enuncian.

Tras el seguimiento radiográfico, el resultado muestra una cicatrización clínica pero no radiológica y eso, nos lleva a pensar y dudar de una falta de neo-
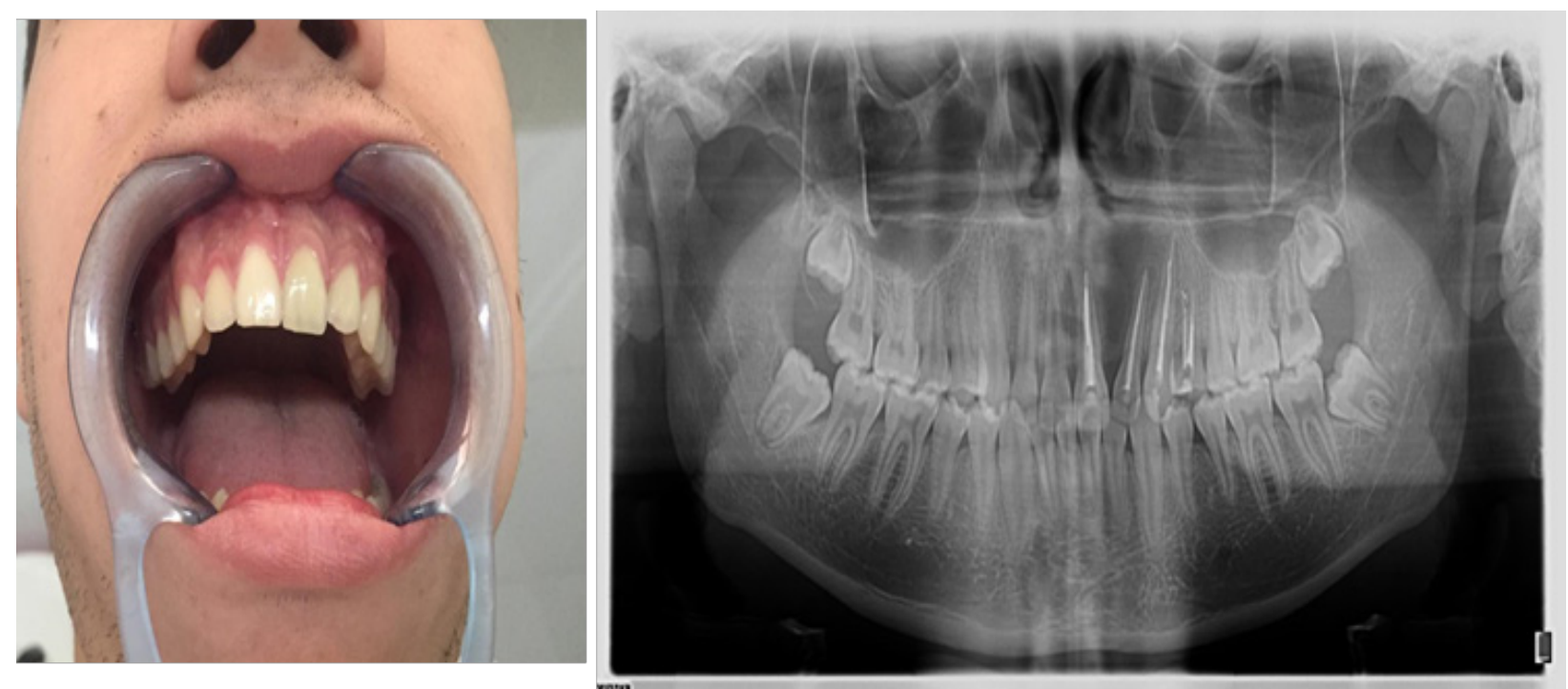

Figura 3. Control clínico y radiográfico postoperatorio con evolución favorable. 
formación ósea, sin embargo, la reosificacion necesita de un periodo entre 6-24 meses. Por esta razón y teniendo en cuenta las probabilidades de recidiva del quiste, se realizó un seguimiento del paciente durante un lapso de 2 años aproximadamente.

Esta patología, tiene ciertas características de un quiste, pero también posee las características de una neoplasia sólida y transformación maligna. Corroboramos con el presente caso clínico la bibliografía consultada, ya que se trató de una lesión unilocular radiolúcida, con expansión y perforación de la tabla vestibular y desplazamiento dentario. Este caso corresponde a Quiste Odontogenico Calcificante de tipo Intraóseo.

El cirujano bucomaxilofacial deberá disponer de exámenes complementarios y coadyuvantes a la biopsia, la Tomografía Cone Beam para la adecuada planificación del tratamiento de lesiones intraóseas que ayuden a visualizarla de manera detallada y la íntima relación con las estructuras anatómicas y a la vez, demarquen la técnica quirúrgica a emplear.

Las indicaciones post operatorias cumplidas, la asistencia del paciente a los controles y el seguimiento de su evolución, además del manejo interdisciplinario son factores que también influyen en el éxito del tratamiento quirúrgico.

\section{Referencias Bibliográficas}

1. Pindborg JJ, Kramer IRH, Torloni H. Clasificación histológica internacional de tumores Nro 5. Tipos histológicos de tumores odontogénicos, quistes maxilares y lesiones afines. Organización Mundial de la Salud (Ginebra). 1972; 1- 144. Disponible: en: https://apps.who.int/iris/bitstream/handle/10665/37798/924376005X_es.pdf;jsessionid

2. León VA, Diaz MR, Miranda HO. Quiste odontogénico calcificante de amplia dimensión: Reporte de caso con 8 meses de acompañamiento. Rev Od Univ Central Ecuador; 2019;21(1):70-79. DOI: 10.29166/odontologia. vol21.n1.2019-69-79

3. Cavalieri-Pereira L, Cavalieri-Pereira R, Gomes Cavalieri-Pereira LGL. Tratamento cirúrgico de cisto odontogênico epitelial calcificante. Rev Bras Cir Craniomaxilofac. 2012;15(2):101-4. Disponible en: https://www.cromp. com.br/pdf/tratamento_cirurgico_de_cisto_odontogenico_epitelial_calcificante.pdf

4. Visconti MAPG, Junqueira RB, Verner FS, Rodrigues AAS, Devito KL, Visconti Filho RF. Tomografía Computarizada de Haz Cónico como Instrumento Complementario de Diagnóstico y Planeamiento Quirúrgico de Quiste Dentígero: Reporte de un Caso. Int J Odontostomat. 2014;8(1):85-91. Disponible en: https://scielo.conicyt.cl/ pdf/ijodontos/v8n1/art11.pdf

5. Morales Trejo B, Carbajal Bello L. Quiste odontogénico calcificante (quiste de Gorlin). Reporte de un caso y su seguimiento. Revisión de la literatura. Rev ADM. 1999; LVI (2):83-87. Disponible: https://www.medigraphic.com/ pdfs/adm/od-1999/od992h.pdf

6. Martínez López M J, Cruz Jimenez T. Tumores odontógenos. [en intenet] Diciembre 2006 [citado Febrero 2010]. Disponible en: URL: http://eusalud.uninet.edu/ apuntes/tema_22.pd 